\title{
Auditory enhancement under simultaneous masking in normal-hearing and hearing-impaired listeners
}

\author{
Heather A. Kreft, ${ }^{\text {a) }}$ Magdalena Wojtczak, and Andrew J. Oxenham \\ Department of Psychology, University of Minnesota, Elliott Hall, 75 East River Parkway, Minneapolis, \\ Minnesota 55455, USA
}

(Received 29 August 2017; revised 9 January 2018; accepted 24 January 2018; published online 13 February 2018)

\begin{abstract}
Auditory enhancement, where a target sound within a masker is rendered more audible by the prior presentation of the masker alone, may play an important role in auditory perception under variable everyday acoustic conditions. Cochlear hearing loss may reduce enhancement effects, potentially contributing to the difficulties experienced by hearing-impaired (HI) individuals in noisy and reverberant environments. However, it remains unknown whether, and by how much, enhancement under simultaneous masking is reduced in $\mathrm{HI}$ listeners. Enhancement of a pure tone under simultaneous masking with a multi-tone masker was measured in $\mathrm{HI}$ listeners and age-matched normalhearing $(\mathrm{NH})$ listeners as function of the spectral notch width of the masker, using stimuli at equal sensation levels as well as at equal sound pressure levels, but with the stimuli presented in noise to the NH listeners to maintain the equal sensation level between listener groups. The results showed that $\mathrm{HI}$ listeners exhibited some enhancement in all conditions. However, even when conditions were made as comparable as possible, in terms of effective spectral notch width and presentation level, the enhancement effect in HI listeners under simultaneous masking was reduced relative to that observed in NH listeners. (C) 2018 Acoustical Society of America.
\end{abstract}

https://doi.org/10.1121/1.5023687

[JJL]

Pages: 901-910

\section{INTRODUCTION}

When a pure-tone or narrowband-noise signal is masked by a broadband sound with a spectral notch around the signal, its detection threshold can be substantially reduced by a preceding copy of the masker alone. This effect is known as auditory enhancement (Viemeister, 1980). The mechanisms underlying auditory enhancement may allow the auditory system to adapt to the acoustic environment by reducing the effect of slow and long-term changes in the acoustic environment. Such mechanisms may therefore assist in achieving perceptual constancy of important sounds, such as speech, despite different talkers, room acoustics, and background noises. The mechanisms underlying enhancement may also assist in making sudden changes in the ongoing acoustic environment more salient, thereby alerting us to the presence of new events.

The original enhancement effect under simultaneous masking could be explained by neural adaptation of the response to the masker by its preceding copy (precursor) leading to an enhancement of the response to the signal, relative to the response to the surrounding masker components. However, a mechanism based on simple adaptation cannot easily explain why enhancement is also observed under forward masking (e.g., Viemeister and Bacon, 1982). In this case a spectrally notched precursor can be shown to increase the masking effectiveness of a following target component in the spectral notch, suggesting that its neural representation has been enhanced in both relative terms (relative to the

a)Electronic mail: plumx002@umn.edu surrounding masker components) and absolute terms (compared to the target's representation in the absence of the precursor). Simple adaptation effects also cannot explain why the loudness of a target in a masker is enhanced by a precursor, whereas the loudness of the masker itself is not affected (Wang and Oxenham, 2016). To explain the available data, a concept known as "adaptation of inhibition" has been postulated (Viemeister and Bacon, 1982). According to this theory, the components of the masker inhibit the response to the target and to one another, but this inhibition effect adapts over time. When the precursor is present, the inhibitory effect of the masker on the target would have been reduced due to this adaptation, leading to a stronger response to the target when it is presented (e.g., Viemeister and Bacon, 1982; Byrne et al., 2011).

Adaptation that occurs at the level of the auditory nerve could contribute to the effect, although it is likely to contribute only to the relative, and not to absolute, enhancement of the target. Effects of lateral ("two-tone") suppression could occur at the level of basilar membrane and auditory nerve (Abbas and Sachs, 1976; Rhode and Cooper, 1993), but these effects are known to be nearly instantaneous, and thus inconsistent with the time constants required to account for perceptual auditory enhancement (Viemeister, 1980; Feng and Oxenham, 2015). On the other hand, efferent effects (Guinan, 2006) could potentially contribute to changing cochlear responses with the appropriate time constants. Evidence against a peripheral locus for enhancement effects comes from a study in humans that failed to find correlates of enhancement in the human cochlea via otoacoustic emissions (Beim et al., 2015) and from a study in the cat auditory 
nerve (Palmer et al., 1995) that found evidence for adaptation but not absolute enhancement. However, neither study can be said to unequivocally rule out cochlear or auditorynerve contributions to enhancement. In the human study (Beim et al., 2015), otoacoustic emissions were measured under passive conditions; it is possible that different results might have been obtained if the participants had been simultaneously performing an active detection task, if one assumes that attention-based efferent activation plays a role. In the case of the auditory-nerve study of Palmer et al. (1995), the cats were anethsetized, again potentially limiting any role of the efferent system.

A study of human envelope following responses (EFR), thought to reflect primarily midbrain responses, found no correlates of enhancement (Carcagno et al., 2014). On the other hand, a single-unit study of responses within the awake marmosets' inferior colliculus (IC) did find neurons that exhibited some key properties of auditory enhancement (Nelson and Young, 2010). This apparent discrepancy may be due to the possibility that the EFR, as a population response, does not sufficiently reflect the responses of the subset of neurons that exhibit enhancement.

Behavioral evidence for a potentially peripheral locus of the effect comes from studies that have found that enhancement effects are much stronger when the preceding masker (or precursor) is presented to the same ear as the target than when it is presented to the opposite ear (e.g., Byrne et al., 2011).

Another way to assess the extent to which peripheral processing is involved in auditory enhancement is to test people with sensorineural hearing loss or with a cochlear implant (CI). If enhancement requires the normal functioning of the cochlear amplifier (including sharp frequency tuning, amplitude compression, and suppression effects), then listeners with typical cochlear hearing loss associated with outer hair cell damage should exhibit reduced or absent enhancement. Similarly, if enhancement requires the normal function of the inner hair cells and synapses, including some properties of adaptation that are absent or altered under electrical stimulation (e.g., Parkins, 1989), then listeners with a CI should not exhibit enhancement. One study of CI users followed a paradigm introduced by Summerfield et al. (1984), and found that a broadband stimulus with spectral gaps corresponding to the formant frequencies of vowels could make the perception of the vowels more likely, suggesting that the spectral gaps led to some enhancement (Wang et al., 2012). The enhancement of vowel formants is similar in nature to the enhancement of a target under simultaneous masking. A study of CI users that examined enhancement under both simultaneous and forward masking found evidence for enhancement under simultaneous masking, but not forward masking (Kreft and Oxenham, 2017). One possible explanation is that the mechanisms yielding relative but not absolute enhancement (reflected in simultaneous masking and vowel perception) are intact and therefore rely on mechanisms more central than the cochlea, whereas the mechanisms yielding absolute enhancement (reflected in forward masking and loudness matching tasks) are not, either because they are peripheral in nature, or because they rely on a peripheral input that is not sufficiently well represented by the CI.

Little is known about auditory enhancement in hearingimpaired (HI) listeners. One study found little or no evidence for enhancement in HI listeners, using both vowel identification and forward masking, suggesting a peripheral locus of enhancement (Thibodeau, 1991). However, several factors make a direct comparison of enhancement in $\mathrm{NH}$ and $\mathrm{HI}$ listeners difficult. First, the amount of enhancement depends to some extent on the width of the spectral gap in the masker (Viemeister et al., 2013). If HI listeners have broader cochlear filters (e.g., Moore et al., 1999), it may be that the previous study did not test enhancement at the optimal spectral gap for $\mathrm{HI}$ listeners, leading to underestimates of enhancement. Second, the amount of enhancement is known to be somewhat level dependent, with little or no enhancement at the very lowest levels in normal-hearing $(\mathrm{NH})$ listeners (Viemeister, 1980; Viemeister et al., 2013). It may therefore be that the lack of strong enhancement effects found in the study of HI listeners was due to the fact that the $\mathrm{HI}$ and $\mathrm{NH}$ listeners were tested at different sensation levels. Third, to our knowledge, there have been no studies of auditory enhancement under simultaneous masking in HI listeners. Thus, it is not known whether HI exhibit any enhancement under simultaneous masking even if, as found by Thibodeau (1991), they exhibit no enhancement under forward masking.

In the current study, auditory enhancement was measured under simultaneous masking in both $\mathrm{HI}$ and agematched NH listeners. Enhancement was measured over a range of spectral notchwidths. Both groups were tested using stimuli presented at the same sensation level (SL). In one condition the stimuli were presented in quiet, and in another condition, the stimuli were presented to the $\mathrm{NH}$ listeners in the presence of a background noise that raised the detection thresholds of the stimuli to simulate the hearing loss of their age-matched HI counterparts. Thus, in this condition, both groups were tested using the same sound pressure level (SPL) and the same SL. This approach of using masking noise to simulate increased absolute thresholds while maintaining high absolute levels has been shown to simulate some but not all of the effects associated with hearing loss (Oxenham and Dau, 2004; Reed et al., 2009; Gregan et al., 2010).

\section{METHODS}

\section{A. Listeners}

Eight HI listeners were tested (5 females, 3 males with sensorineural hearing loss of unknown origin; average age $=68.5 \mathrm{yr}$ ), along with eight age-matched $\mathrm{NH}$ listeners (6 females, 2 males; average age $=66.9 \mathrm{yr})$. The HI listeners had four-frequency pure tone average (4F-PTA; 500, 1000, 2000, and $4000 \mathrm{~Hz}$ ) between 25 and $60 \mathrm{~dB}$ hearing level (HL) in the test ear. The age-matched controls had audiometric thresholds of $20 \mathrm{~dB} \mathrm{HL}$ or better at octave frequencies from 250 to $4000 \mathrm{~Hz}$ in the test ear. An Interacoustics Equinox 2.0 PCbased audiometer (Interacoustics A/S, Denmark) was used to obtain a standard audiogram for all subjects, and the results for 


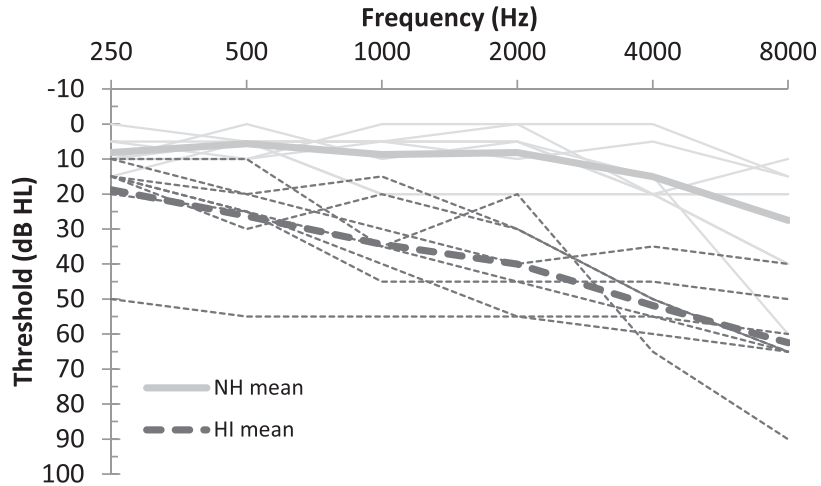

FIG. 1. Audiometric thresholds (in $\mathrm{dB}$ HL) as a function of frequency for normal-hearing (solid lines) and hearing-impaired (dotted lines) listeners. The average audiometric thresholds for each group of listeners are also shown. Listeners were designated as normal hearing if their audiometric thresholds were $20 \mathrm{~dB}$ HL or less for octave frequencies between 250 and $4000 \mathrm{~Hz}$.

each test ear are shown in Fig. 1. The average audiometric thresholds for the HI listeners are displayed by the bold dashed line. They ranged from normal hearing at $250 \mathrm{~Hz}$ to a moderate sensorineural hearing loss at $8000 \mathrm{~Hz}$. The left ear was tested in all subjects, except for one HI listener, who was tested in the right ear. The HI listeners displayed symmetric hearing losses (threshold difference $\leq 10 \mathrm{~dB}$ ) at all test frequencies, except for a few instances in which the difference between thresholds in the two ears was between 10 and $20 \mathrm{~dB}$. In these cases, the ear with the poorer thresholds was the test ear for the entire experiment. All experimental protocols were approved by the Institutional Review Board of the University of Minnesota, all listeners provided informed written consent prior to participation, and all listeners were compensated for their time.

\section{B. Stimuli}

The precursor stimuli were $500 \mathrm{~ms}$ in duration, while the masker and target stimuli were $100 \mathrm{~ms}$ in duration, including 10-ms raised-cosine onset and offset ramps. The gap duration between the offset of the precursor (when present) and the onset of the masker-target complex was $20 \mathrm{~ms}$. The target frequency was 1,2 , or $4 \mathrm{kHz}$. For each target, the masker consisted of four logarithmically spaced sinusoids, geometrically centered at the target frequency. The spectral notch width was set to $0.6,0.9$, or 1.2 octaves. The frequencies of the components for the different notch widths and target frequencies are shown in Table I. These notch widths were selected to include the notch width of 0.6 octaves that produced the maximum amount of enhancement in NH listeners under comparable conditions (Viemeister et al., 2013) as well as wider notches ( 0.9 and 1.2 octaves) to test whether poorer frequency selectivity among the HI listeners might lead to wider notches producing maximum enhancement. In the main enhancement condition (ENH), the precursor consisted of the same four logarithmically spaced tones as the masker. In the condition with no precursor (MSK), only the masker was presented. In the third condition (CON), the precursor contained both the masker and target components ( 5 components total); see Fig. 2. The third condition was added, as it has served as a control condition in other studies, where the presence of a precursor might improve performance, irrespective of any enhancement effects (Carcagno et al., 2012; Feng and Oxenham, 2015). In all cases, the level per component of the precursor was the same as that of the masker.

The stimuli were presented in quiet and in the presence of a threshold-equalizing noise (TEN) (Moore et al., 2000). The TEN provides thresholds for sinusoidal targets that are approximately independent of the target frequency. The purpose of the noise was to elevate the target thresholds for the $\mathrm{NH}$ listeners to approximate those obtained for the individual HI listeners to allow for comparisons at equal SPL. The TEN was bandpass filtered between 20 and $8000 \mathrm{~Hz}$.

\section{Procedure}

The stimuli were generated and processed using Matlab (Mathworks, Natick, MA) via a 24-bit digital-to-analog converter (Lynx22, Lynx Studio Technology, Costa Mesa, CA) and were presented monaurally via headphones (HD650, Sennheiser, Old Lyme, CT). The listeners were seated individually in a double-walled sound-attenuating booth.

First, the absolute thresholds for a 100-ms 1 -, 2 -, and 4$\mathrm{kHz}$ sinusoid were measured for each listener. The thresholds were measured using a three-interval, three-alternative forced-choice (3IFC/3AFC) task with a two-down, one-up adaptive procedure that tracks the $70.7 \%$-correct point on the psychometric function (Levitt, 1971), corresponding to a $d^{\prime}$ value of 1.26. Correct-answer feedback was provided after each trial. The initial signal level was set to be easily detectable; the initial step size of the tracking procedure was $8 \mathrm{~dB}$. After two reversals in the direction of the tracking procedure, the step size was reduced to $4 \mathrm{~dB}$ for another two reversals, and then was reduced to the final step size of $2 \mathrm{~dB}$. The mean target level at the last eight reversal points was defined as the threshold for the run, and the estimates from three adaptive runs were averaged to obtain a final threshold value for each frequency and listener.

In addition, for the NH listeners only, masked thresholds were measured for the same 1-, 2-, or 4-kHz sinusoid in the presence of TEN. Each NH listener was paired with a HI listener, and the TEN level was set to simulate the hearing loss

TABLE I. Frequencies $(\mathrm{Hz})$ of the four maskers for each target frequency and notch width.

\begin{tabular}{lccc}
\hline \hline & & Notch width & \\
\cline { 2 - 4 } Target frequency $(\mathrm{Hz})$ & 0.6 octaves & 0.9 octaves & 1.2 octaves \\
\hline 1000 & $757.9,812.3,1231.1,1319.5$ & $683.0,732.0,1366.0,1464.1$ & $615.6,659.8,1515.7,1624.5$ \\
2000 & $1515.7,1624.5,2462.3,2639.0$ & $1366.0,1464.1,2732.1,2928.1$ & $1231.1,1319.5,3031.4,3249.0$ \\
4000 & $3031.4,3249.0,4924.6,5278.0$ & $2732.1,2928.1,5464.2,5856.4$ & $2462.3,2639.0,6062.9,6498.0$ \\
\hline \hline
\end{tabular}



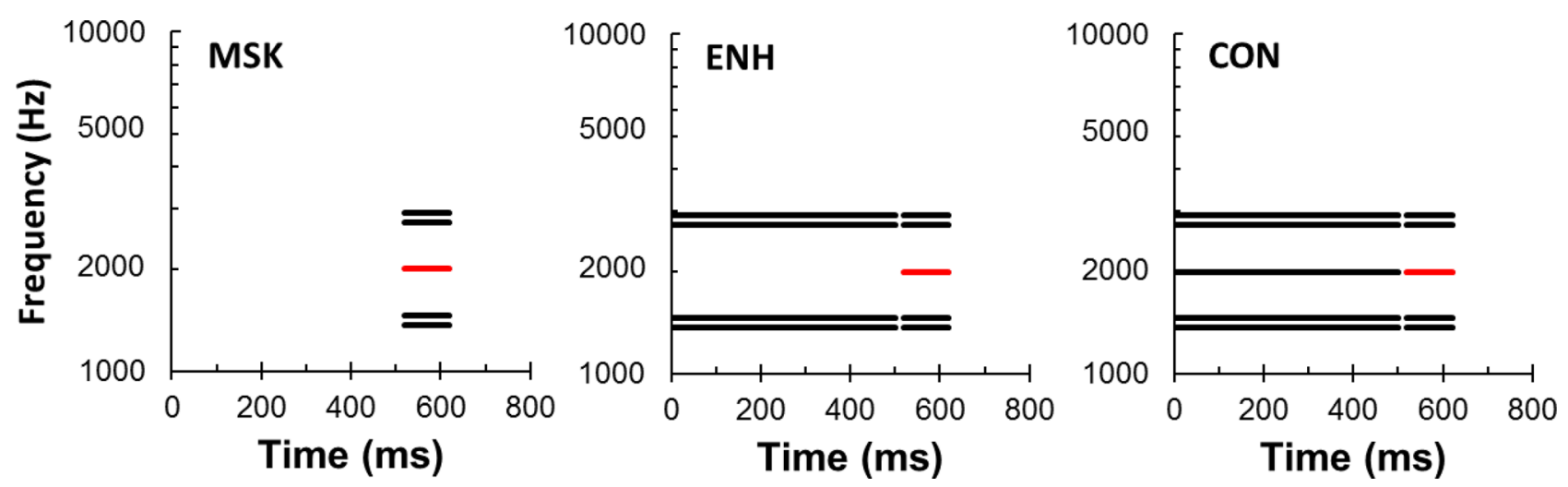

FIG. 2. (Color online) Schematic diagrams of the masking conditions tested in this study. The condition with the masker only (MSK) involves just the masker and the target. The enhanced condition (ENH) includes the precursor without the target component, leading to expected enhancement of the target. The control condition (CON) also has the precursor, but with the target component present, leading to no expectation of enhancement despite the presence of a precursor. The experiments involved a two-interval, two-alternative forced-choice task; here only the intervals containing the target are shown.

for that corresponding HI listener at each target frequency. For instance, if the HI listener's threshold for the target at $1 \mathrm{kHz}$ was $45 \mathrm{~dB}$ SPL, then the TEN level was set to $45 \mathrm{~dB}$ SPL per equivalent rectangular bandwidth (ERB) around $1 \mathrm{kHz}$ for the paired NH listener. The procedure used to measure thresholds in TEN for each target frequency was the same as described above. When present, the TEN noise was gated on $100 \mathrm{~ms}$ prior to the start of the first interval and gated off $100 \mathrm{~ms}$ after the end of the last interval in each trial.

For each target frequency, the absolute thresholds (without and with TEN for the NH group) were used to set the level per component of the masker in the simultaneous masking enhancement task. For the HI group, the masker level per component was set to $20 \mathrm{~dB}$ above the absolute target threshold. For the NH group, two masker levels were tested. The first was also $20 \mathrm{~dB}$ per masker component above the average absolute threshold for that listener (equal SL condition). The second corresponded to $20 \mathrm{~dB}$ above the average threshold in the presence of the TEN, which on average was equal to the SPL used for the matched HI listener $(63.1 \mathrm{~dB}$ SPL for the NH group and 63.5 dB SPL for HI group) (equal SPL condition).

In the main experiment, once the masker and precursor levels were determined, the target threshold was measured in the presence of the masker alone (MSK), or in the presence of the masker and precursor, where the precursor was either a copy of the masker (ENH), or it also included the target component (CON); see Fig. 2. A two-interval, two-alternative forced choice (2IFC/2AFC) task was used with a twodown one-up adaptive tracking procedure, corresponding to a $d^{\prime}$ value of 0.77 , with the same step sizes and number of reversals as described above for the measurement of absolute threshold. A final threshold for each condition (ENH, MSK, and $\mathrm{CON}$ ) was determined by averaging three (or five, if standard deviation of first three estimates was greater than $4 \mathrm{~dB}$ ) estimates for each frequency and notch width for each listener. To calculate the enhancement effect, as defined traditionally for simultaneous masking $\left(\mathrm{EE}_{\mathrm{sim} 1}\right)$, the target threshold in the ENH condition was subtracted from that in the MSK condition. An alternative definition of enhancement (e.g., Carcagno et al., 2012; Feng and Oxenham, 2015) was obtained by subtracting target threshold in the ENH condition from that in the CON condition $\left(E_{\mathrm{sim} 2}\right)$. This alternative method is a useful control if the precursor, even with the target component present, results in improved performance relative to a no-precursor condition.

\section{RESULTS}

Figure 3 shows the average masked thresholds in $\mathrm{dB}$ SPL for the three conditions tested (MSK, CON, and ENH). The left, middle, and right columns present data from the 1-, $2-$, and 4-kHz target, respectively. The top row presents data from the HI listeners, the middle row presents data from the $\mathrm{NH}$ listeners at equal SL, and the bottom row presents data from the NH listeners at equal SPL in the presence of TEN. In each panel, the average threshold for the target alone in quiet (or in TEN) is shown by the horizontal dashed line.

The general pattern of results is similar across all frequencies and all listener groups: thresholds in the CON condition tend to be highest, followed by thresholds in the MSK condition, and thresholds in the ENH condition tend to be lowest. Thresholds also tend to decrease with increasing notch width across all conditions.

Figure 4 replots the results in terms of target-to-masker ratio $(\mathrm{dB})$, defined as the target level relative to masker level per component. A negative target-to-masker ratio implies that the target component was lower in level than the individual masker components. In most cases, for both the $\mathrm{NH}$ and HI listeners, target-to-masker ratios were negative. For most target levels at threshold, the overall increase in stimulus level due to the target would have been likely below threshold for detecting an increase in the overall level of the target-plus-masker complex over that for the masker alone. This implies that listeners were able to "hear out" the target and were not relying on overall changes in the stimulus level.

Figure 5 replots the results in terms of amount of enhancement, defined either as the difference in threshold between the ENH and MSK conditions ( $\mathrm{EE}_{\text {sim } 1}$; left column) or as the difference in threshold between the ENH and CON conditions ( $\mathrm{EE}_{\mathrm{sim} 2}$; right column). Averaged across target 
$1000 \mathrm{~Hz}$

HI
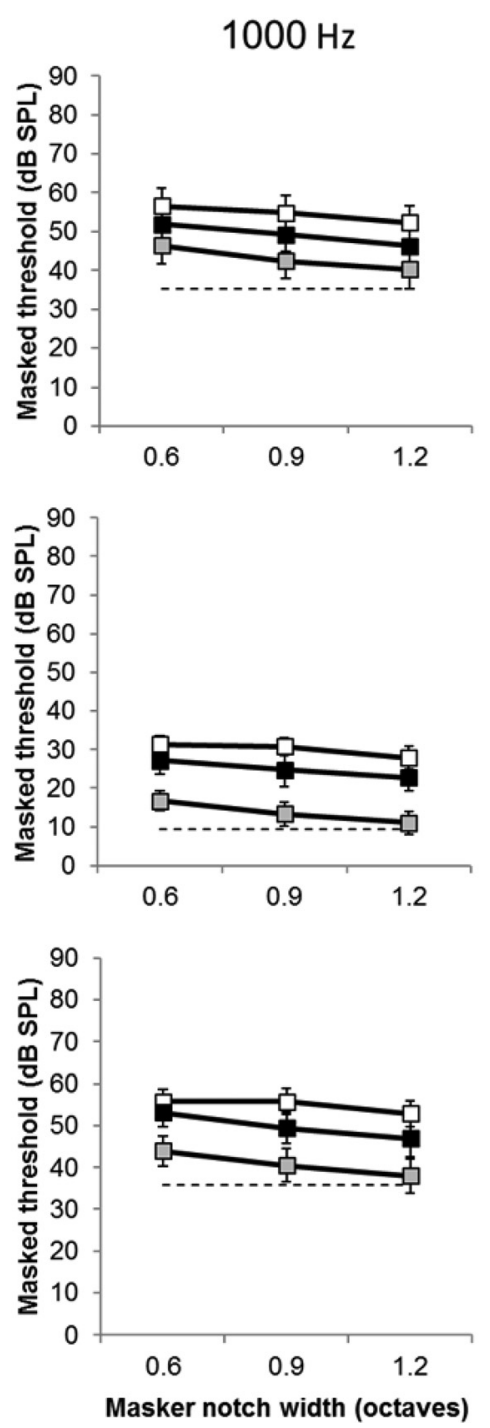

$2000 \mathrm{~Hz}$
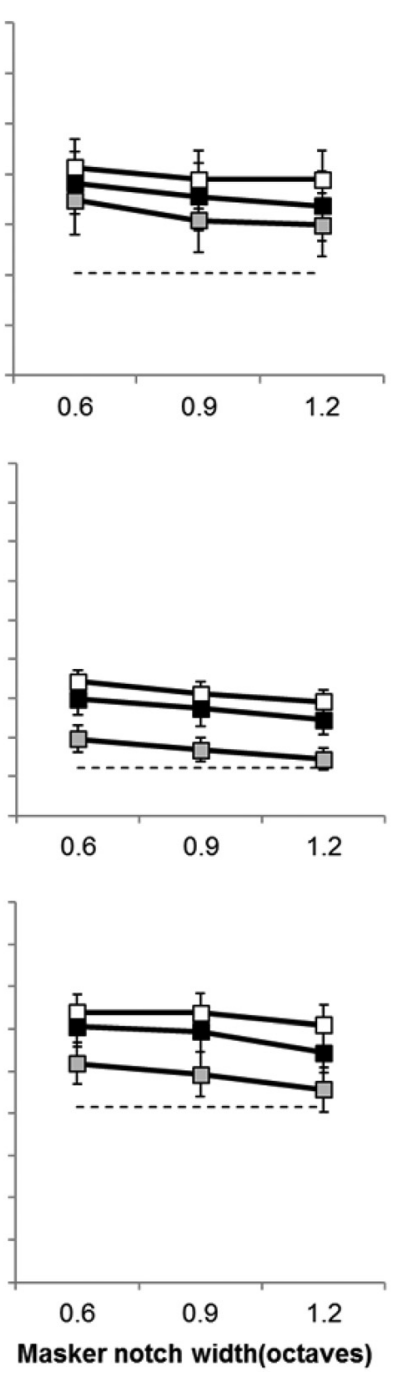

$4000 \mathrm{~Hz}$
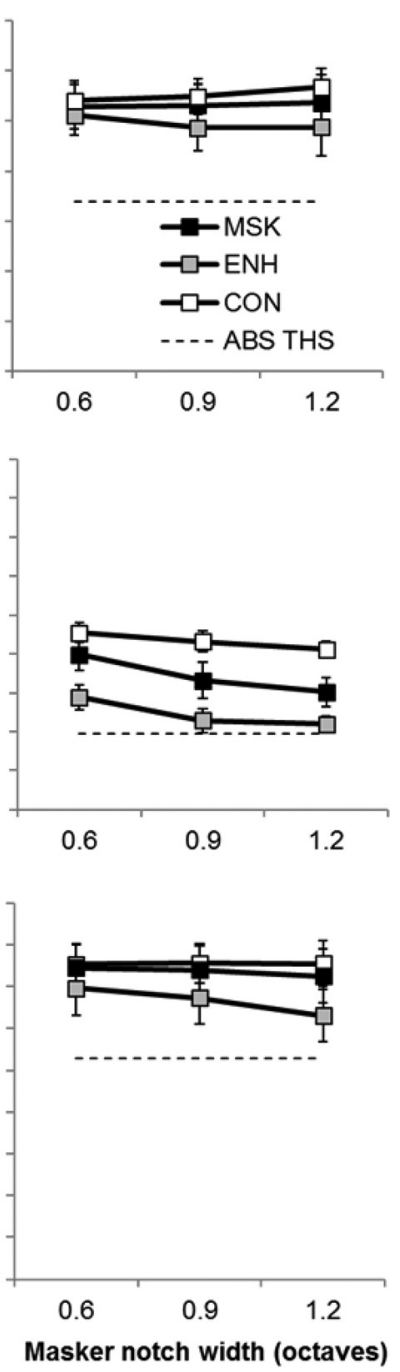

FIG. 3. Group averages for thresholds of each condition as a function of the spectral notch width of the masker. The top row shows results from the HI group. The middle row shows results from the NH group with the stimuli presented in quiet at roughly the same SL as for the HI group. The bottom row shows results from the NH group with the stimuli presented in a background of TEN to simulate the hearing loss of the matched HI listener for each NH listener and with the masker and enhancer presented at the same SPL as for the HI listeners. Each column shows data using a different signal frequency (1, 2, and $4 \mathrm{kHz}$, from left to right). Error bars represent 1 standard error of the mean across listeners.

frequencies and notch widths, the amount of enhancement $\left(\mathrm{EE}_{\mathrm{sim} 1}\right)$ was $10.5,8.4$, and $4.4 \mathrm{~dB}$ for the $\mathrm{NH}$ equal $\mathrm{SL}, \mathrm{NH}$ equal SPL, and HI groups, respectively. Compared with the $\mathrm{CON}$ condition $\left(\mathrm{EE}_{\mathrm{sim} 2}\right)$, the average amount of enhancement was $16.5,12.3$, and $8.5 \mathrm{~dB}$, for the $\mathrm{NH}$ equal $\mathrm{SL}, \mathrm{NH}$ equal SPL, and HI groups, respectively.

To determine whether stimulus level (and/or the presence of TEN) affected enhancement in NH listeners, a repeatedmeasures analysis of variance (ANOVA) was performed with enhancement as the dependent variable, enhancement measure $\left(\mathrm{EE}_{\mathrm{sim} 1}\right.$ or $\left.\mathrm{EE}_{\mathrm{sim} 2}\right)$, target frequency $(1,2$, or $4 \mathrm{kHz})$, and notch width $(0.6,0.9$, or 1.2 octaves) as within-subjects factors. Here, and in all subsequent ANOVAs, a Huynh-Feldt correction was applied as appropriate for any violations of sphericity. The results showed significant main effects of stimulus level $\left(\mathrm{F}_{1,7}=12.0, \mathrm{p}=0.011\right.$, partial $\left.\mathrm{p}^{2}=0.631\right)$ and enhancement measure $\left(\mathrm{F}_{1,7}=14.7, \mathrm{p}=0.006\right.$, partial $\mathrm{y}^{2}=0.677$ ), as well as a significant two-way interaction between stimulus level and enhancement measure $\left(\mathrm{F}_{1,7}=5.88, \mathrm{p}=0.046\right.$, partial $\left.\mathrm{y}^{2}=0.457\right)$, and a significant three-way interaction between stimulus level, enhancement measure, and notch width $\left(\mathrm{F}_{1.44,10.11}=8.42, \mathrm{p}=0.010\right.$, partial $\left.\mathrm{n}^{2}=0.546\right)$. The effect of frequency was not significant, nor was any interaction with frequency ( $p>0.05$ in all cases). The main effect of stimulus level reflects the fact that enhancement tends to be larger for the equal-SL condition than for the equal-SPL condition (in TEN). The main effect of enhancement measure reflects the fact that the $\mathrm{EE}_{\mathrm{sim} 2}$ measure tended to result in higher levels of enhancement than the $\mathrm{EE}_{\text {sim1 }}$ measure, perhaps because of some residual forward masking effects by the precursor component at the target frequency of the target itself in the CON conditions. The interaction between level and enhancement measure, as well as the three-way interaction, reflects the stronger effect of level for the $\mathrm{EE}_{\mathrm{sim} 2}$ measure than for the $\mathrm{EE}_{\mathrm{sim} 1}$ measure, particularly at the widest notch width. 
$1000 \mathrm{~Hz}$

HI

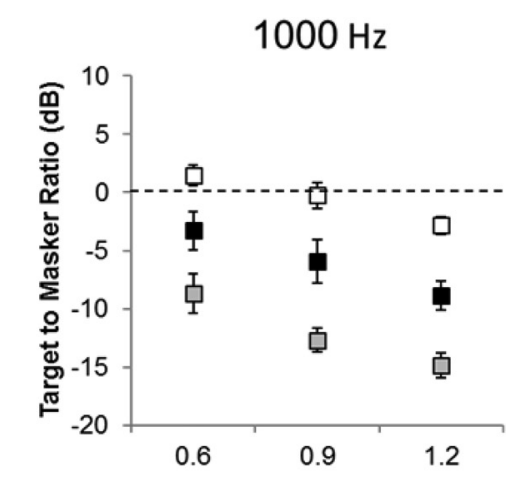

NH - equal SL

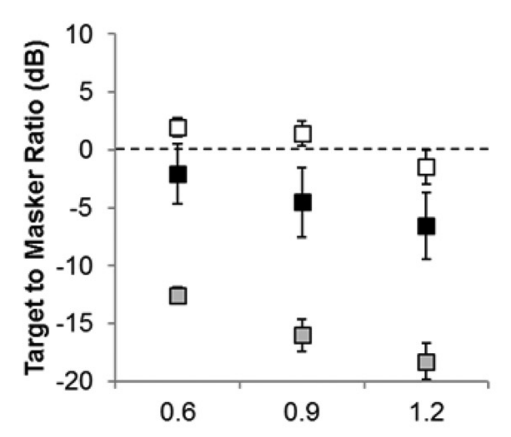

NH - equal SPL

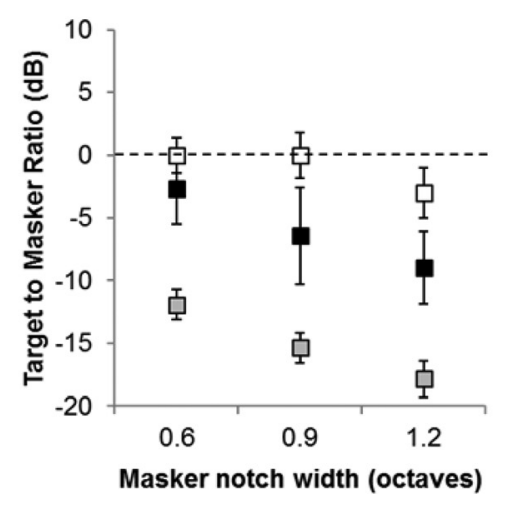

$2000 \mathrm{~Hz}$
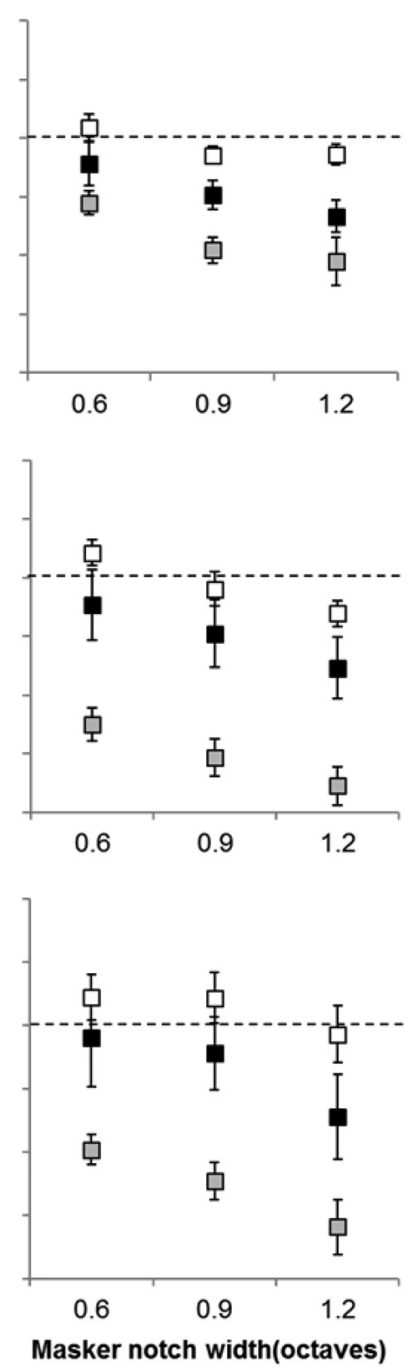

$4000 \mathrm{~Hz}$
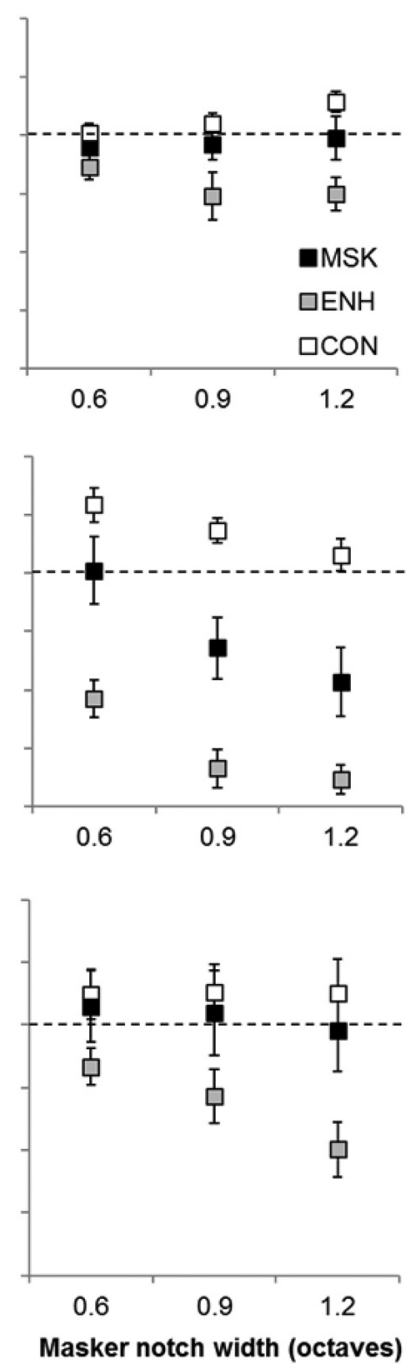

FIG. 4. Target thresholds, replotted from Fig. 3 in terms of target-to-masker ratio (in dB). The horizontal zero dB lines represent the target level at which it is equal to the level of each of the masker components.

To determine whether hearing status ( $\mathrm{NH}$ versus $\mathrm{HI}$ ) affected enhancement at equal SL, a repeated-measures analysis of variance (ANOVA) was performed with the data from the HI listeners and from the NH listeners at equal SL in quiet. The amount of enhancement was the dependent variable, enhancement measure $\left(\mathrm{EE}_{\mathrm{sim} 1}\right.$ or $\left.\mathrm{EE}_{\mathrm{sim} 2}\right)$, target frequency $(1,2$, or $4 \mathrm{kHz})$, and notch width $(0.6,0.9$, or 1.2 octaves) were within-subjects factors, and hearing status was a between-subjects factor. The results showed significant main effects of enhancement measure $\left(\mathrm{F}_{1,14}=44.7\right.$, $\mathrm{p}<0.001$, partial $\left.\mathrm{p}^{2}=0.762\right)$, and hearing status $\left(\mathrm{F}_{1,14}=104.7, \mathrm{p}<0.001\right.$, partial $\left.\mathrm{p}^{2}=0.882\right)$. There were significant two-way interactions between enhancement measure and target frequency $\left(\mathrm{F}_{2,28}=7.4, \mathrm{p}=0.003\right.$, partial $\left.\mathrm{y}^{2}=0.346\right)$, and notch width and hearing status $\left(\mathrm{F}_{2,28}=3.5\right.$, $\mathrm{p}=0.045$, partial $\left.\mathrm{p}^{2}=0.198\right)$, as well as a significant threeway interaction between enhancement measure, notch width, and hearing status $\left(\mathrm{F}_{2,28}=4.5, \quad \mathrm{p}=0.021\right.$, partial $\left.\mathrm{y}^{2}=0.241\right)$. No other main effects or interactions reached significance.
To determine whether hearing status ( $\mathrm{NH}$ versus $\mathrm{HI}$ ) affected enhancement at equal SPL, a repeated-measures analysis of variance (ANOVA) was performed with the data from the HI listeners and from the NH listeners at equal SPL in the presence of noise. The amount of enhancement was the dependent variable, enhancement measure $\left(\mathrm{EE}_{\mathrm{sim} 1}\right.$ or $\left.\mathrm{EE}_{\text {sim2 }}\right)$, target frequency $(1,2$, or $4 \mathrm{kHz})$, and notch width $(0.6,0.9$, or 1.2 octaves) were within-subjects factors and hearing status was a between-subjects factor. The results showed significant main effects of enhancement measure $\left(\mathrm{F}_{1,14}=27.7, \mathrm{p}<0.001\right.$, partial $\left.\mathrm{j}^{2}=0.664\right)$, notch width $\left(\mathrm{F}_{2,28}=4.76, \mathrm{p}=0.017\right.$, partial $\left.\mathrm{\eta}^{2}=0.254\right)$, and hearing status $\left(\mathrm{F}_{1,14}=10.2, \mathrm{p}=0.006\right.$, partial $\left.\mathrm{p}^{2}=0.422\right)$. There were significant two-way interactions between enhancement measure and notch width $\left(\mathrm{F}_{2,28}=10.4, \mathrm{p}<0.001\right.$, partial $\left.\mathrm{y}^{2}=0.427\right)$ and between enhancement measure and target frequency $\left(\mathrm{F}_{1.76,24.69}=10.9, \mathrm{p}=0.001\right.$, partial $\left.\mathrm{p}^{2}=0.438\right)$. No other main effects or interactions reached significance.

Considering both the equal-SL and equal-SPL comparisons, the results show that $\mathrm{HI}$ listeners exhibited less 

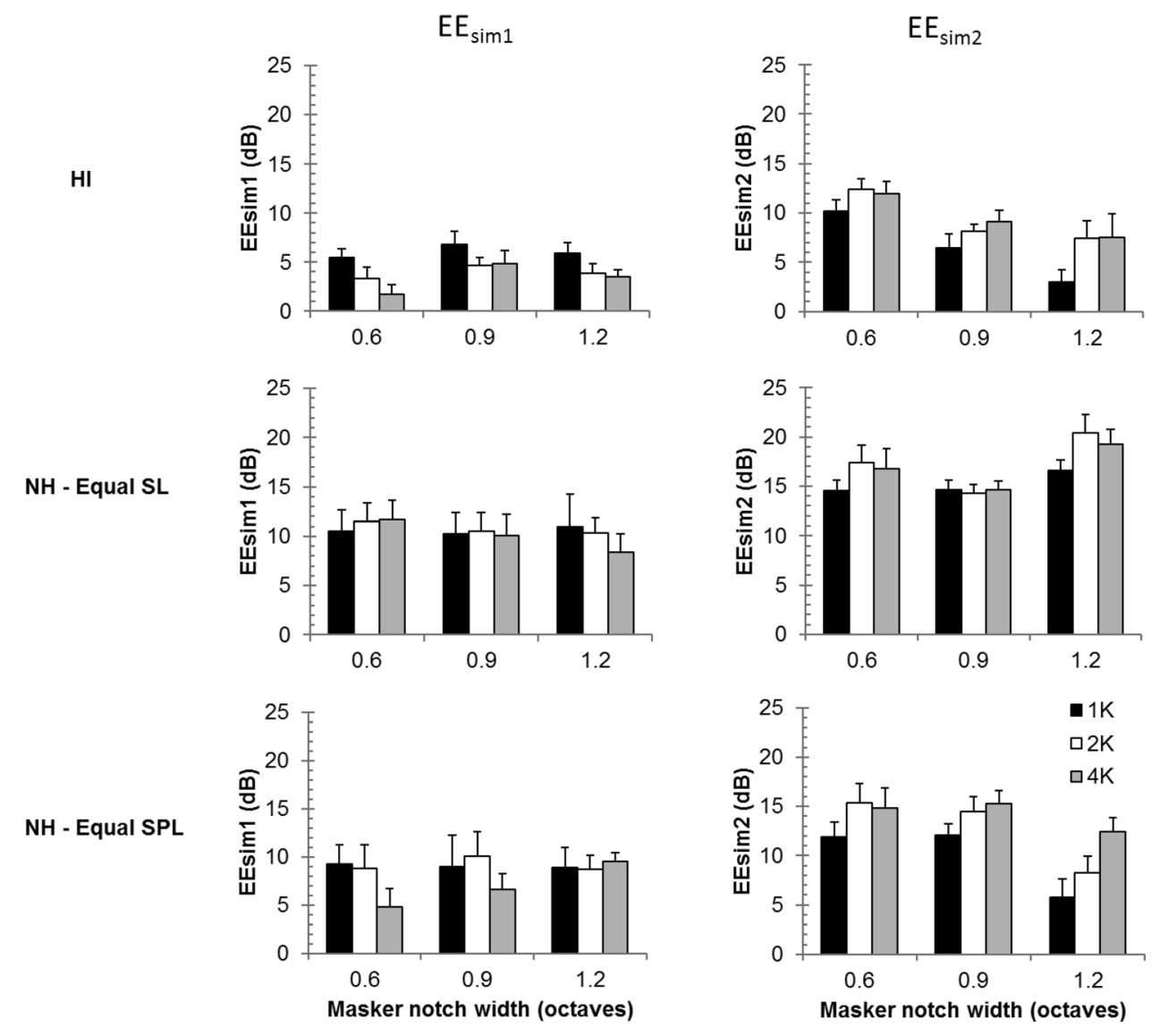

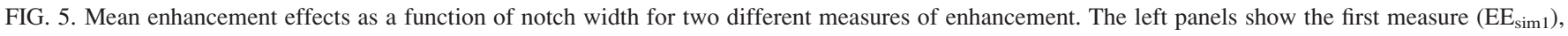
obtained by subtracting thresholds in the $\mathrm{ENH}$ condition from thresholds in the MSK condition (in $\mathrm{dB}$ ). The right panel shows the second measure (EE $\mathrm{sim}_{2}$ ), which is derived by subtracting thresholds in the ENH condition from thresholds in the CON condition (in $\mathrm{dB}$ ). The top row shows results from the HI group. The middle row shows results from the NH group with the stimuli presented in quiet at the same SL as for the HI group. The bottom row shows results from the NH group with the stimuli presented in a background of TEN to simulate the hearing loss of the matched HI listener for each NH listener and with the masker and precursor presented at the same SPL as for the HI listeners. Error bars represent 1 standard error of the mean.

enhancement than NH listeners at both stimulus levels tested, at all frequencies, and regardless of which enhancement measure was used. Although a marginally significant $(\mathrm{p}=0.045)$ interaction between hearing loss and notch width was observed when comparing equal-SL conditions, the fact that no other interactions with hearing loss were observed suggests that hearing loss resulted in an overall reduction in enhancement across all conditions.

Given the significant group effect on enhancement measures, we next considered whether the amount of hearing loss affected the amount of enhancement observed. This comparison is by necessity preliminary in nature, given the small number (8) of HI listeners tested here. Nevertheless, our power was increased by the fact that enhancement was measured at three signal frequencies. Because absolute thresholds at the three frequencies were different within individual listeners, we treated thresholds at the three frequencies as independent samples, resulting in 24 data points from the HI listeners. As shown in Fig. 6, there appears to be some relationship between the amount of enhancement ( $\mathrm{EE}_{\text {sim } 1}$ in $\mathrm{dB}$, averaged across the three notch widths), and thresholds in quiet at each frequency. The Pearson product moment correlation coefficient just reached significance $(\mathrm{r}=-0.408, \mathrm{p}=0.048)$.

\section{DISCUSSION}

Comparing the conditions with and without a precursor $\left(\mathrm{EE}_{\mathrm{sim} 1}\right)$, and pooling across target frequency and notch width, the average amount of enhancement was 4.4, 8.4, and $10.5 \mathrm{~dB}$ for the $\mathrm{HI}$, NH equal SPL, and NH equal SL groups, respectively. The average amount of enhancement observed in both NH conditions of between 8.4 and $10.5 \mathrm{~dB}$ is generally consistent with that found in earlier studies. For instance, Viemeister (1980) found individual enhancement of between 6.7 and $13.6 \mathrm{~dB}$ for targets at 1 and $3 \mathrm{kHz}$ and maskers that were either harmonic complex tones or wideband noise with a spectral notch around the target frequency. Also using a spectrally notched noise around the target frequency of $1 \mathrm{kHz}$, Carlyon (1989) found maximum enhancement for individual subjects of between 5.9 and $16.7 \mathrm{~dB}$, again averaging around $10 \mathrm{~dB}$. More recently, Viemeister et al. (2013) reported mean enhancement levels of around $5 \mathrm{~dB}$, considerably less than found here and in previous studies. Given that they used maskers (harmonic, inharmonic, and noise), notch widths, and masker levels that were similar to those used here and in previous studies, it is not clear why their average reported enhancement levels were lower. 


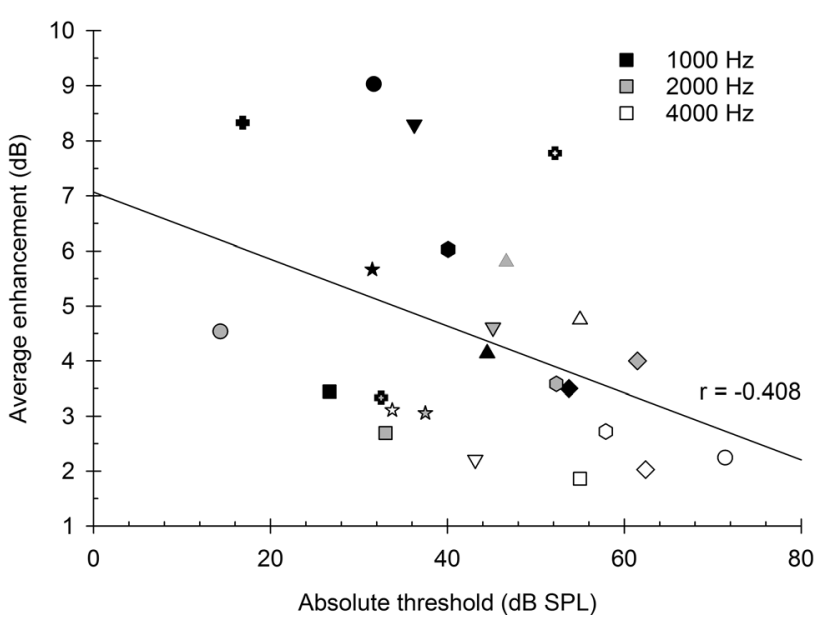

FIG. 6. Mean enhancement effects (averaged across notch widths) as a function of quiet adaptive thresholds for each HI listener. The fill color of the symbols is different for each target frequency $(1000 \mathrm{~Hz}$-black, $2000 \mathrm{~Hz}-$ gray, and $4000 \mathrm{~Hz}$ - white), and each symbol type represents a different $\mathrm{HI}$ listener. The dashed line shows the regression line, suggesting a significant relationship between absolute threshold and amount of enhancement $(\mathrm{r}=0.408, \mathrm{p}=0.048)$.

To our knowledge, enhancement in HI listeners under simultaneous masking has not been reported previously. Our results suggest some, but reduced, enhancement in HI listeners. Although many aspects of the effect of hearing loss can be ascribed to the use of higher physical sound levels and/or reduced audibility for HI listeners (Reed et al., 2009), other aspects cannot (Oxenham and Dau, 2004; Gregan et al., 2010). In this case, the reduced enhancement, relative to our NH controls, cannot be ascribed to differences in either absolute level or sensation level, as both were controlled in the comparison NH equal SPL group, where audibility was restricted by the use of a TEN masker.

Our study is also the first to test enhancement in older groups of both NH and HI listeners. The fact that the maximum amount of enhancement in the $\mathrm{NH}$ group was similar to that reported in earlier studies with younger listeners (e.g., Viemeister, 1980; Carlyon, 1989; Reed et al., 2009) suggests that age does not play a major role in determining the amount of enhancement.

If not absolute level or audibility, then what explains the reduced enhancement found in HI listeners? One possible explanation tested here was that enhancement is present but requires a larger spectral notch to be observed. Such an explanation is rendered possible by the fact that frequency selectivity is generally degraded by cochlear hearing loss, presumably due to the loss of outer hair cell function (e.g., Moore and Oxenham, 1998; Moore et al., 1999; (Oxenham and Bacon, 2003). Our data do not support this explanation: the amount of enhancement was not strongly dependent on notch width for either the NH or HI listeners, and there was no indication that the amount of enhancement reached a maximum at a wider notch width for the HI listeners than for the NH listeners.

As our HI listeners had at least moderate hearing loss at some or all of the test frequencies, it is likely that they suffered from some degree of loss of outer hair cell function, leading to a loss of gain and a loss of basilar membrane compression (Ruggero and Rich, 1991; Oxenham and Plack, 1997; Oxenham and Bacon, 2003). The loss of compression in particular may affect the amount of enhancement measured. For example, an enhancement effect that increases the effective representation of the target by $2 \mathrm{~dB}$ may require a change of $10 \mathrm{~dB}$ in the acoustic level of the target to overcome the effects of basilar membrane compression, which has been estimated to be as much as 5:1 (Ruggero, 1992; Oxenham and Plack, 1997; Moore et al., 1999). A reduction in compression, due to loss of outer hair cell function, would result in a reduction in the change of the acoustic target level, even if the "internal" effects of enhancement remain unchanged. This mechanism may explain the apparent reduction in enhancement under simultaneous masking observed here. It is possible that the effective compression in the $\mathrm{NH}$ group was reduced in the equal-SL condition, as the target levels were generally quite low (between 10 and $45 \mathrm{~dB}$ SPL), where the response of the basilar membrane may be more linear (Ruggero, 1992; Oxenham and Plack, 1997). The fact that enhancement was similar for the NH listeners in both the equal-SL and equal-SPL conditions suggests that compression did not play a major role. Alternatively, compression may have also been reduced somewhat in the equal-SPL condition by the presence of the background noise (Recio-Spinoso and Lopez-Poveda, 2010), although not all studies have found such effects (Gregan et al., 2010).

Another possible explanation is that enhancement under simultaneous masking is the combination of at least two processes. One, proposed by Carlyon (1989), involves higherlevel processes, such as perceptual grouping, whereby the precursor forms a single perceptual stream with the masker, and so assists in the perceptual segregation of the target from the masker. Although other studies have argued against a purely central explanation of enhancement (Hicks and Bacon, 1992; Byrne et al., 2011; Viemeister et al., 2013), it remains a possible contributing factor. Another involves more peripheral processing, such as suppression or lateral inhibition, which directly influences the neural representation of the target at a relatively early stage in auditory processing. It may be that the more central processes are intact in listeners with cochlear hearing loss, but that the peripheral contributions are reduced, leading to less enhancement overall.

Hearing loss may also involve a loss of inner hair cell function and/or loss of synaptic connections between the inner hair cells and the auditory nerve (Kujawa and Liberman, 2009). The perceptual effects of such loss remain uncertain in humans (e.g., Oxenham, 2016; Plack et al., 2016), and it is unclear whether or how it would affect enhancement. If a synaptic loss results in reduced efferent activation, and if enhancement relies on efferent stimulation, then such a loss could also affect enhancement. It is worth noting, however, that ageing, which may result in a loss of synapses without measurable hearing loss (Ruggles et al., 2012; Sergeyenko et al., 2013), does not seem to affect enhancement, given that our older $\mathrm{NH}$ group seemed to show as much enhancement as found in earlier studies with younger NH listeners. 
A comparison of our results with those of Thibodeau (1991) suggests that HI listeners experience enhancement under simultaneous masking but not under forward masking. Before drawing this conclusion, it should be noted that Thibodeau's participants had more hearing loss on average than those tested here, with average thresholds of about $57 \mathrm{~dB}$ HL at $2 \mathrm{kHz}$. However, those HI listeners in the current study with the most hearing loss (furthest right symbols in Fig. 6), with thresholds between 50 and $70 \mathrm{~dB}$ HL, still showed some residual enhancement, suggesting that differences in degree of hearing loss cannot account for the different outcomes of the two studies. The finding of enhancement in simultaneous but not forward masking is consistent with the recent findings in CI users (Kreft and Oxenham, 2017), again suggesting the possibility of two mechanisms, with only one affected by cochlear hearing loss. A similar twomechanism explanation was recently proposed by Wang et al. $(2015,2016)$ to account for differences between CI users and $\mathrm{NH}$ listeners in tasks involving loudness context effects. The nature of these two mechanisms remains unknown. One may be central in nature, relying on grouping mechanisms, as suggested by Carlyon (1989), whereas the other may be more peripheral, involving mechanisms that are affected by hearing loss or cochlear implantation, such as decreased cochlear gain, reduced suppression, and/or reduced efferent effects, as discussed above. Another alternative is that both mechanisms are relatively peripheral (at least precortical), but that one relies on mechanisms (such as adaptation in the inferior colliculus) that are not directly affected by hearing loss.

In summary, auditory enhancement was observed under simultaneous masking in HI listeners, although the amount of enhancement was reduced relative to $\mathrm{NH}$ listeners, even when matched for age, overall level, and audibility. Taken together with earlier data suggesting that HI listeners experience no enhancement under forward masking, the results suggest that enhancement may involve at least two mechanisms, at least one of which is affected by cochlear hearing loss.

\section{ACKNOWLEDGMENTS}

This research was supported by NIDCD Grant No. R01 DC 012262. The authors wish to extend special thanks to the subjects who participated in this study. The authors declare that they have no conflict of interest.

Abbas, P. J., and Sachs, M. B. (1976). "Two-tone suppression in auditorynerve fibers: Extension of a stimulus-response relationship," J. Acoust. Soc. Am. 59, 112-122.

Beim, J. A., Elliott, M., Oxenham, A. J., and Wojtczak, M. (2015). "Stimulus frequency otoacoustic emissions provide no evidence for the role of efferents in the enhancement effect," J. Assoc. Res. Otolaryngol. 16, 613-629.

Byrne, A. J., Stellmack, M. A., and Viemeister, N. F. (2011). "The enhancement effect: Evidence for adaptation of inhibition using a binaural centering task," J. Acoust. Soc. Am. 129, 2088-2094.

Carcagno, S., Plack, C. J., Portron, A., Semal, C., and Demany, L. (2014).

"The auditory enhancement effect is not reflected in the $80-\mathrm{Hz}$ auditory steady-state response," J. Assoc. Res. Otolaryngol. 15, 621-630.
Carcagno, S., Semal, C., and Demany, L. (2012). "Auditory enhancement of increments in spectral amplitude stems from more than one source," J. Assoc. Res. Otolaryngol. 13, 693-702.

Carlyon, R. P. (1989). "Changes in the masked thresholds of brief tones produced by prior bursts of noise," Hear. Res. 41, 223-236.

Feng, L., and Oxenham, A. J. (2015). "New perspectives on the measurement and time course of auditory enhancement," J. Exp. Psychol. Hum. Percept. Perform. 41, 1696-1708.

Gregan, M. J., Nelson, P. B., and Oxenham, A. J. (2010). "Effects of background noise level on behavioral estimates of basilar-membrane compression," J. Acoust. Soc. Am. 127, 3018-3025.

Guinan, J. J., Jr. (2006). "Olivocochlear efferents: Anatomy, physiology, function, and the measurement of efferent effects in humans," Ear Hear. 27, 589-607.

Hicks, M. L., and Bacon, S. P. (1992). "Factors influencing temporal effects with notched-noise maskers," Hear. Res. 64, 123-132.

Kreft, H. A., and Oxenham, A. J. (2017). "Auditory enhancement in cochlear-implant users under simultaneous and forward masking," J. Assoc. Res. Otolaryngol. 18, 483-493.

Kujawa, S. G., and Liberman, M. C. (2009). "Adding insult to injury: Cochlear nerve degeneration after 'temporary' noise-induced hearing loss," J. Neurosci. 29, 14077-14085.

Levitt, H. (1971). "Transformed up-down methods in psychoacoustics," J. Acoust. Soc. Am. 49, 467-477.

Moore, B. C. J., Huss, M., Vickers, D. A., Glasberg, B. R., and Alcantara, J. I. (2000). "A test for the diagnosis of dead regions in the cochlea," Br. J. Audiol. 34, 205-224.

Moore, B. C. J., and Oxenham, A. J. (1998). "Psychoacoustic consequences of compression in the peripheral auditory system," Psychol. Rev. 105, $108-124$.

Moore, B. C. J., Vickers, D. A., Plack, C. J., and Oxenham, A. J. (1999). "Inter-relationship between different psychoacoustic measures assumed to be related to the cochlear active mechanism," J. Acoust. Soc. Am. 106, 2761-2778.

Nelson, P. C., and Young, E. D. (2010). "Neural correlates of contextdependent perceptual enhancement in the inferior colliculus," J. Neurosci. 30, 6577-6587.

Oxenham, A. J. (2016). "Predicting the perceptual consequences of hidden hearing loss," Trends Hear. 20, 1-6.

Oxenham, A. J., and Bacon, S. P. (2003). "Cochlear compression: Perceptual measures and implications for normal and impaired hearing," Ear Hear. 24, 352-366.

Oxenham, A. J., and Dau, T. (2004). "Masker phase effects in normalhearing and hearing-impaired listeners: Evidence for peripheral compression at low signal frequencies," J. Acoust. Soc. Am. 116, 2248-2257.

Oxenham, A. J., and Plack, C. J. (1997). "A behavioral measure of basilarmembrane nonlinearity in listeners with normal and impaired hearing," J. Acoust. Soc. Am. 101, 3666-3675.

Palmer, A. R., Summerfield, Q., and Fantini, D. A. (1995). "Responses of auditory-nerve fibers to stimuli producing psychophysical enhancement," J. Acoust. Soc. Am. 97, 1786-1799.

Parkins, C. W. (1989). "Temporal response patterns of auditory nerve fibers to electrical stimulation in deafened squirrel monkeys," Hear. Res. 41, $137-168$.

Plack, C. J., Leger, A., Prendergast, G., Kluk, K., Guest, H., and Munro, K. J. (2016). "Toward a diagnostic test for hidden hearing loss," Trends Hear. 20, 1-9.

Recio-Spinoso, A., and Lopez-Poveda, E. A. (2010). "Basilar membrane responses to simultaneous presentations of white noise and a single tone," in The Neurophysiological Bases of Auditory Perception, edited by E. A. Lopez-Poveda, A. R. Palmer, and R. Meddis (Spinger Verlag, New York,), pp. 15-23.

Reed, C. M., Braida, L. D., and Zurek, P. M. (2009). "Review article: Review of the literature on temporal resolution in listeners with cochlear hearing impairment: A critical assessment of the role of suprathreshold deficits," Trends Amplif. 13, 4-43.

Rhode, W. S., and Cooper, N. P. (1993). "Two-tone suppression and distortion production on the basilar membrane in the hook region of the cat and guinea pig cochleae," Hear. Res. 66, 31-45.

Ruggero, M. A. (1992). "Responses to sound of the basilar membrane of the mammalian cochlea," Curr. Opin. Neurobiol. 2, 449-456.

Ruggero, M. A., and Rich, N. C. (1991). "Furosemide alters organ of Corti mechanics: Evidence for feedback of outer hair cells upon the basilar membrane," J. Neurosci. 11, 1057-1067. 
Ruggles, D., Bharadwaj, H., and Shinn-Cunningham, B. G. (2012). "Why middle-aged listeners have trouble hearing in everyday settings," Curr. Biol. 22, 1417-1422.

Sergeyenko, Y., Lall, K., Liberman, M. C., and Kujawa, S. G. (2013). “Agerelated cochlear synaptopathy: An early-onset contributor to auditory functional decline," J. Neurosci. 33, 13686-13694.

Summerfield, Q., Haggard, M. P., Foster, J., and Gray, S. (1984). "Perceiving vowels from uniform spectra: Phonetic exploration of an auditory after-effect," Percept. Psychophys. 35, 203-213.

Thibodeau, L. M. (1991). "Performance of hearing-impaired persons on auditory enhancement tasks," J. Acoust. Soc. Am. 89, 2843-2850.

Viemeister, N. F. (1980). "Adaptation of masking," in Psychophysical, Physiological and Behavioural Studies in Hearing, edited by G. van den Brink and F. A. Bilsen (Delft U.P., Delft, Netherlands), pp. $190-198$.
Viemeister, N. F., and Bacon, S. P. (1982). "Forward masking by enhanced components in harmonic complexes," J. Acoust. Soc. Am. 71, 1502-1507.

Viemeister, N. F., Byrne, A. J., and Stellmack, M. A. (2013). "Spectral and level effects in auditory signal enhancement," Adv. Exp. Med. Biol. 787, 167-174.

Wang, N., Kreft, H., and Oxenham, A. J. (2012). "Vowel enhancement effects in cochlear-implant users," J. Acoust. Soc. Am. 131, EL421-EL426.

Wang, N., Kreft, H. A., and Oxenham, A. J. (2015). "Loudness context effects in normal-hearing listeners and cochlear-implant users," J. Assoc. Res. Otolaryngol. 16, 535-545.

Wang, N., Kreft, H., and Oxenham, A. J. (2016). "Induced loudness reduction and enhancement in acoustic and electric hearing," J. Assoc. Res. Otolaryngol. 17, 383-391.

Wang, N., and Oxenham, A. J. (2016). "Effects of auditory enhancement on the loudness of masker and target components," Hear. Res. 333, 150-156. 